\title{
HAT inhibitor, garcinol, exacerbates lipopolysaccharide-induced inflammation in vitro and in vivo
}

\author{
BIN WANG ${ }^{1}$, LING LIN ${ }^{2}$, QING AI $^{3}$, TAO ZENG $^{4}$, PU GE $^{1}$ and LI ZHANG $^{2,5}$ \\ ${ }^{1}$ Department of Anesthesiology, The First Affiliated Hospital; Departments of ${ }^{2}$ Pathophysiology and ${ }^{3}$ Physiology, \\ Chongqing Medical University, Chongqing 400016; ${ }^{4}$ Medical College, Jingchu University of Technology, \\ Jingmen, Hubei 448000; ${ }^{5}$ Laboratory of Stem Cell and Tissue Engineering, \\ Chongqing Medical University, Chongqing 400016, P.R. China
}

Received July 21, 2015; Accepted April 18, 2016

DOI: $10.3892 / \mathrm{mmr} .2016 .5189$

\begin{abstract}
Acetylation modification catalyzed by histone acetyl transferases (HATs) is important for transcriptional regulation. The present study investigated the effects of the HAT inhibitor garcinol on the expression of inflammation-associated genes in lipopolysaccharide (LPS)-stimulated RAW264.7 murine macrophages and LPS-challenged mice. The levels of pro-inflammatory cytokines were determined by reverse transcription-quantitative polymerase chain reaction and enzyme-linked immunosorbent assay. The degree of multi-organ injury was evaluated by histopathological examination of the lung, determination of the alanine aminotransferase and blood urea nitrogen in plasma samples and by monitoring the survival rate of the experimental animals. The results of the current study demonstrated that garcinol promoted LPS-induced expression of tumor necrosis factor- $\alpha$ (TNF- $\alpha$ ) and interleukin-6 (IL-6) in RAW264.7 cells. These effects were associated with reduced acetylation of nuclear factor- $\mathrm{\kappa B}$ p65. Additionally, treatment with garcinol enhanced LPS-induced expression of TNF- $\alpha$ and IL-6, exacerbated LPS-induced lung injury, increased LPS-induced elevation of plasma alanine aminotransferase and blood urea nitrogen, and reduced the survival rate of LPS-challenged mice. These data indicated that the HAT inhibitor, garcinol, enhances LPS-induced inflammation in vitro and in vivo, suggesting that acetylation modification has an important regulatory function during inflammation.
\end{abstract}

\footnotetext{
Correspondence to: Dr Li Zhang, Department of Pathophysiology, Chongqing MedicalUniversity, 1 Yixueyuan Road,Chongqing 400016, P.R. China

E-mail: zhangli@cqmu.edu.cn
}

Key words: histone acetyltransferase, garcinol, lipopolysaccharide, acetylation, inflammation

\section{Introduction}

Inflammation is a series of coordinated defensive responses against a diverse range of pathogenic insults, which is driven by the programmed release of inflammatory mediators (1). The sequential expression of inflammation-associated genes is tightly regulated at multiple checkpoints, including various post-translational modifications (2). Acetylation is an important post-translational modification of histones, determining the structure and function of chromatin (3). Acetylation of lysines generally results in neutralization of the positive charge on histone tails, weakening the electrostatic interaction between the histone and the DNA backbone, which has been associated with opened structure of chromatin, accessibility for transcription factors and eventually, transcriptional activation (4).

In addition to histones, several non-histone proteins are targets of acetylation modification and their function can be modified following acetylation. For example, mitogen-activated protein kinase (MAPK) phosphatase-1 (MKP-1) can be acetylated on a lysine residue within its substrate-binding domain, which may lead to enhanced interaction with p38, thus, increasing its phosphatase activity and interrupting MAPK signaling (5). Additionally, nuclear factor- $\kappa \mathrm{B}(\mathrm{NF}-\kappa \mathrm{B})$, the pivotal transcription factor that controls the expression of numerous inflammatory mediators, is also acetylated at various lysine residues (6). Acetylation modifications have been previously reported to positively and negatively regulate the subcellular location, DNA binding affinity and transcriptional activity of $\mathrm{NF}-\kappa \mathrm{B}(6)$. Thus, acetylation modification may markedly alter the function of various proteins involved in the expression of inflammation-associated genes (7).

Two groups of enzymes, histone acetyltransferases (HATs) and histone deacetylases (HDACs), have been previously demonstrated to maintain the delicate dynamic equilibrium of the acetylation level of histone lysine residues (8). HDACs have been demonstrated to be important for the inflammatory response and HDAC inhibitors are emerging as promising reagents for the treatment of inflammatory disease (9). However, the pharmacological effects of HAT inhibitors on the inflammatory response remain to be fully elucidated. In the present study, the potential pharmacological effects of garcinol, a commonly used HAT inhibitor $(10,11)$, on the expression of inflammatory 
Table I. Primers used for reverse transcription-quantitative polymerase chain reaction.

\begin{tabular}{lll}
\hline Target gene & \multicolumn{1}{c}{ Forward primer } & Reverse primer \\
\hline Tumor necrosis factor- $\alpha$ & 5-CCAGGTTCTCTTCAAGGGACAA-3 & 5-ACGGCAGAGAGGAGGTTGACT-3 \\
Interleukin-6 & 5-AGTTGCCTTCTTGGGACTGATG-3 & 5-TCTCATTTCCACGATTTCCCAG-3 \\
$\beta$-actin & 5-CTGAGAGGGAAATCGTGCGT-3 & 5-CCACAGGATTCCATACCCAAGA-3
\end{tabular}

genes was investigated in vitro using LPS-stimulated murine RAW264.7 macrophages. Furthermore, garcinol was administrated into mice with LPS-induced endotoxemia and the effects on the plasma pro-inflammatory cytokine level, organ inflammation and injury, and survival were determined. The present study may indicate the potential modulatory effects of HAT inhibitors on inflammation.

\section{Materials and methods}

Reagents. Garcinol and lipopolysaccharide (LPS; from Escherichia coli;, 055:B5) were purchased from Sigma-Aldrich (St. Louis, MO, USA). Alanine aminotransferase (ALT) and blood urea nitrogen (BUN) assay kits were purchased from Nanjing Jiancheng Bioengineering Institute (Nanjing, China). Monoclonal rabbit anti-mouse p65 (cat. no. 8242) and monoclonal rabbit anti-mouse acetyl-p65 (Lys310; cat. no. 12629) antibodies were purchased from Cell Signaling Technology, Inc. (Danvers, MA, USA). Biotinylated goat anti-rabbit antibody (cat. no. 14709) was obtained from Pierce Biotechnology, Inc. (Rockford, IL, USA). The enzyme-linked immunosorbent assay (ELISA) kits for detecting mouse tumor necrosis factor- $\alpha$ (TNF- $\alpha$ ) and interleukin-6 (IL-6) were purchased from NeoBioscience Technology Company (Shenzhen, China).

Animals. Male BALB/c mice ( $\mathrm{n}=112$; age, 6-8 weeks; weight, 20-25 g) were obtained from the Experimental Animal Center of Chongqing Medical University (Chongqing, China). The animals were fed with a standard laboratory diet and water ad libitum. They were housed in a specific-pathogen free room at a temperature of $20-25^{\circ} \mathrm{C}, 50 \pm 5 \%$ relative humidity under a 12-h light/dark cycle. All experimental procedures involving animals were approved by the Animal Care and Use Committee of Chongqing Medical University.

LPS-induced lethal inflammation. Mice were intraperitoneally injected with LPS $(20 \mathrm{mg} / \mathrm{kg})$ to induce lethal inflammation. Dimethyl sulfoxide (Beijing Dingguo Changsheng Biotechnology Co., Ltd., Beijing, China) as a vehicle and garcinol (10 $\mathrm{mg} / \mathrm{kg}$, intraperitoneally) were administrated $0.5 \mathrm{~h}$ prior to LPS challenge. The mice were sacrificed at $18 \mathrm{~h}(\mathrm{n}=8$ per group) subsequent to LPS challenge by anesthesia with pentobarbital $(50 \mathrm{mg} / \mathrm{kg}$; Shanghai Xinya Pharmaceutical Corporation, Shanghai, China). Blood samples and lung tissues were harvested for further experiments. To determine the effect of garcinol on the outcome of lethal inflammation, another group of animals $(n=20)$ were used and the lethality was evaluated every $6 \mathrm{~h}$ for a minimum of 7 days. Surviving mice were sacrificed on the fourteenth day subsequent to LPS exposure by pentobarbital anesthesia as above.
Histological analysis. Formalin-fixed (Chengdu KeLong Chemical Co., Ltd., Chengdu, China) lung specimens were embedded in paraffin (Chengdu KeLong Chemical Co., Ltd.) and stained with hematoxylin and eosin (Nanjing Jiancheng Bioengineering Institute) routinely for conventional morphological examination under a light microscope (BX43; Olympus Corporation, Tokyo, Japan).

ALT and BUN detection. To evaluate the degree of liver and renal injuries, the plasma levels of ALT and BUN were determined with the corresponding detection kits according to the manufacturer's instructions.

Detection of pro-inflammatory cytokines. The concentrations of TNF- $\alpha$ and IL- 6 in plasma samples or cell culture supernatants were determined using the corresponding ELISA kits according to the manufacturer's instructions. Briefly, samples or standards were pipetted into a microplate pre-coated with a monoclonal antibody specific for mouse TNF- $\alpha /$ IL- 6 . The TNF- $\alpha /$ IL- 6 present in the samples was bound by the pre-coated antibody. Following a washing step, an enzyme-linked polyclonal antibody specific for mouse TNF- $\alpha /$ IL- 6 was added to the wells. Following a wash to remove any unbound antibody-enzyme reagent, a substrate solution was added to the wells. The enzyme reaction generated a blue product that turned yellow when the stop solution was added. The intensity of the color was measured at $450 \mathrm{~nm}$ (Varioskan LUX; Thermo Fisher Scientific, Inc., Waltham, MA, USA) and the sample values were calculated according to the standard curve.

Reverse transcription-quantitative polymerase chain reaction $(R T-q P C R)$. The mRNA levels of TNF- $\alpha$ and IL- 6 in lung tissue were determined by RT-qPCR. Briefly, total RNA was isolated from lung samples using TRIzol reagent (Takara Biotechnology Co., Ltd., Dalian, China). DNase I (Takara Biotechnology Co., Ltd.) was used for genomic DNA digestion prior to RT-qPCR. First-strand complementary DNA (cDNA) was synthesized using oligo-dT primers (Takara Biotechnology Co., Ltd.) and the M-MLV reverse transcriptase (Takara Biotechnology Co., Ltd.)in PrimeScript II buffer (Takara Biotechnology Co., Ltd.). qPCR was performed using SYBR green PCR Master mix (Takara Biotechnology Co., Ltd.) with the following cycling conditions $(n=35)$ on a CFX Connect (Bio-Rad Laboratories, Inc., Hercules, CA, USA): Denaturation, $95^{\circ} \mathrm{C}$ for $10 \mathrm{sec}$; annealing, $58^{\circ} \mathrm{C}$ for $20 \mathrm{sec}$; and elongation, $72^{\circ} \mathrm{C}$ for $20 \mathrm{sec}$. The mRNA levels of TNF- $\alpha$ and IL-6 were normalized to the levels of $\beta$-actin and quantified using the $\Delta \mathrm{C}_{\mathrm{T}}$-method (12). The primers used are presented in Table I. 

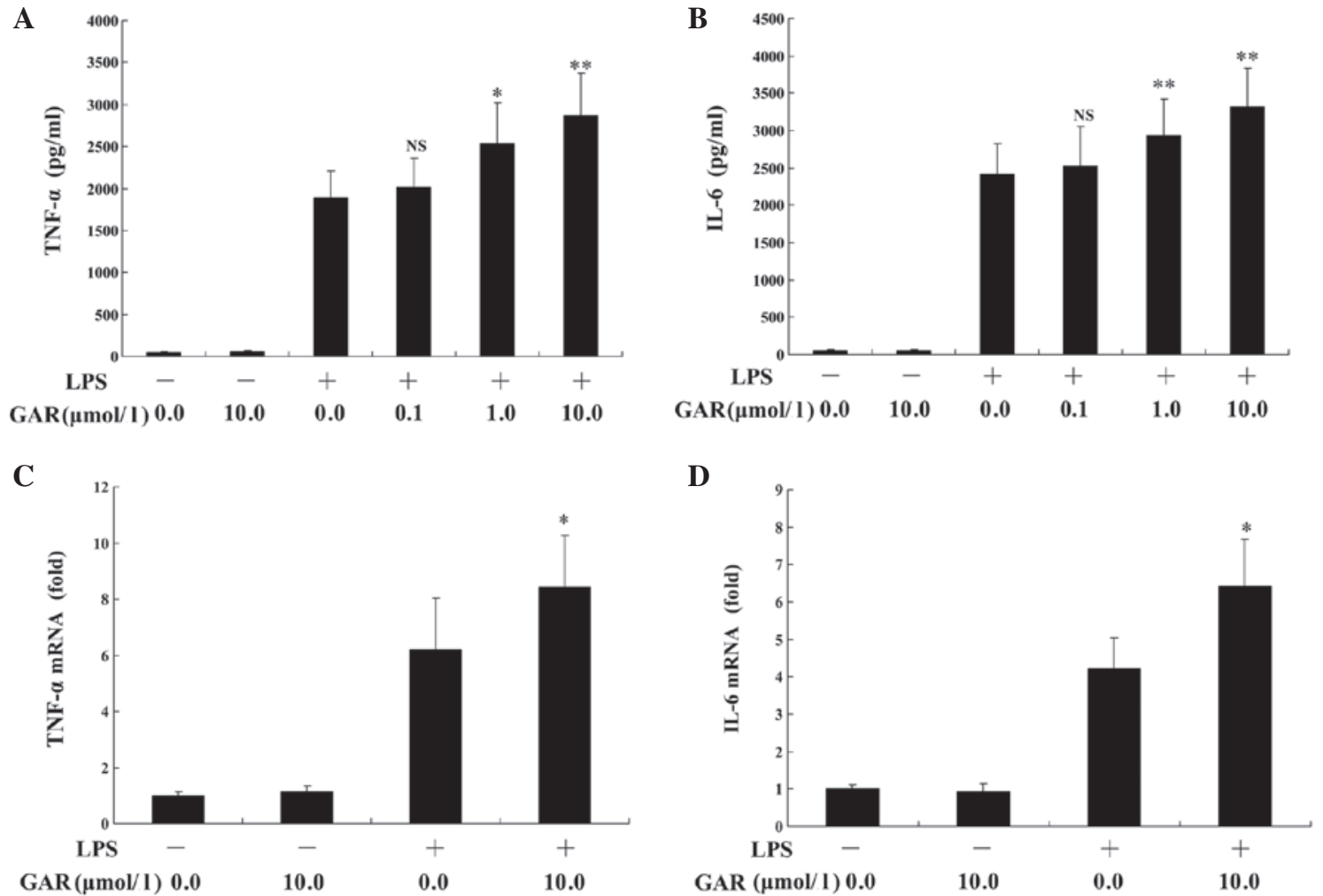

Figure 1. GAR promotes LPS-induced expression of TNF- $\alpha$ and IL-6 in RAW264.7 macrophages. RAW264.7 cells were incubated with vehicle or the indicated doses of garcinol $(0.1,1$ and $10 \mu \mathrm{mol} / 1)$ in the presence or absence of LPS $(100 \mu \mathrm{g} / \mathrm{l})$. The supernatants and cells were harvested after $18 \mathrm{~h}$. The protein levels of (A) TNF- $\alpha$ and (B) IL-6 in the supernatants were determined by enzyme-linked immunosorbent assay. The mRNA levels of (C) TNF- $\alpha$ and (D) IL-6 in cells were determined by reverse transcription-quantitative polymerase chain reaction. Data were presented as the mean \pm standard deviation, $\mathrm{n}=8$. NS, $\mathrm{P}>0.05$ ${ }^{*} \mathrm{P}<0.05,{ }^{* *} \mathrm{P}<0.01$ vs. LPS+/GAR 0 group. GAR, garcinol; LPS, lipopolysaccharide; TNF- $\alpha$, tumor necrosis factor- $\alpha$; IL-6, interleukin-6; NS, non-significant.

Western blot analysis. Total protein lysates from frozen liver samples were prepared according to the method described by Cell Lysis Buffer for Western and IP (Beyotime Institute of Biotechnology, Haimen, China). The total protein concentration was determined using a Pierce BCA Protein assay kit (Thermo Fisher Scientific, Inc.). Protein extracts $(40 \mu \mathrm{g})$ were fractionated on $10 \%$ polyacrylamide-sodium dodecyl sulfate gel (Beyotime Institute of Biotechnology) at $100 \mathrm{~V}$ for $90 \mathrm{~min}$, and then transferred to a nitrocellulose membrane (EMD Millipore, Billerica, MA, USA). The membrane was blocked with 5\% (w/v) non-fat milk in Tris-buffered saline (Beijing Dingguo Changsheng Biotechnology Co., Ltd.) containing 0.05\% Tween-20 (Beijing Dingguo Changsheng Biotechnology Co., Ltd.), and then the membrane was incubated with the primary antibodies (all diluted 1:1,000) overnight at $4^{\circ} \mathrm{C}$, followed by incubation with the secondary antibody (dilution, 1:5,000). Antibody binding was visualized with an enhanced chemiluminescence system (Pierce ECL Western Blotting Substrate; Thermo Fisher Scientific, Inc.) and short exposure of the membrane to X-ray films (Kodak, Rochester, NY, USA). The results were visualized using ChemiDoc Touch Imaging system (Bio-Rad Laboratories, Inc.).

Cell culture and morphological observation. RAW264.7 cells obtained from the Type Culture Collection of the Chinese Academy of Sciences (Shanghai, China) were cultured at $37^{\circ} \mathrm{C}$ in a $5 \% \mathrm{CO}_{2}$ atmosphere humidified incubator, and maintained in RPMI 1640 culture medium (Hyclone; GE

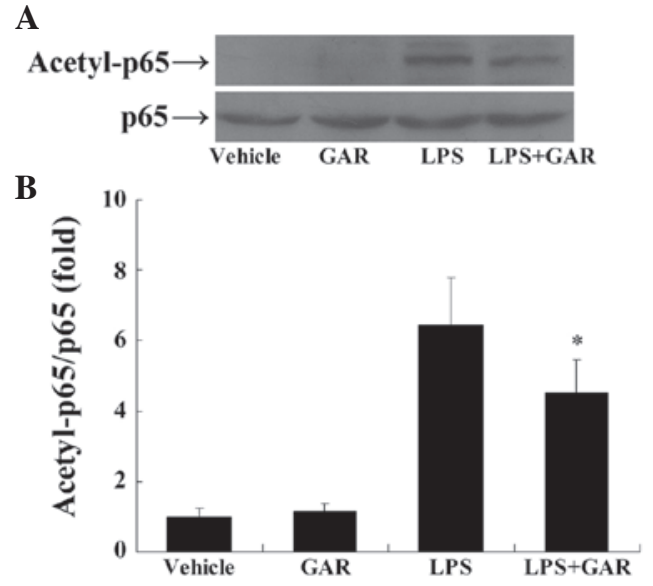

Figure 2. GAR suppresses LPS-induced acetylation of nuclear factor- $\kappa \mathrm{B}$ p65 in RAW264.7 macrophages. RAW264.7 cells were incubated with vehicle or $10 \mu \mathrm{mol} / 1 \mathrm{GAR}$ in the presence or absence of $100 \mu \mathrm{g} / 1 \mathrm{LPS}$. The cells were harvested $18 \mathrm{~h}$ subsequent to LPS challenge. (A) Protein levels of acetylated p65 (Lys310) and total p65 were determined by western blot analysis. The bands of acetylated p65 and p65 are indicated by arrows. (B) Densitometry was performed and data are presented as relative intensity units. Data are expressed as the mean \pm standard deviation, $n=4$. ${ }^{*} \mathrm{P}<0.05$ vs. LPS group. GAR, garcinol; LPS, lipopolysaccharide.

Healthcare Life Sciences, Logan, UT, Utah) containing 10\% heat-inactivated fetal bovine serum (Gibco; Thermo Fisher Scientific, Inc.). RAW264.7 cells were incubated with vehicle or garcinol $(10 \mu \mathrm{mol} / \mathrm{l})$ in the presence or absence of LPS 
A
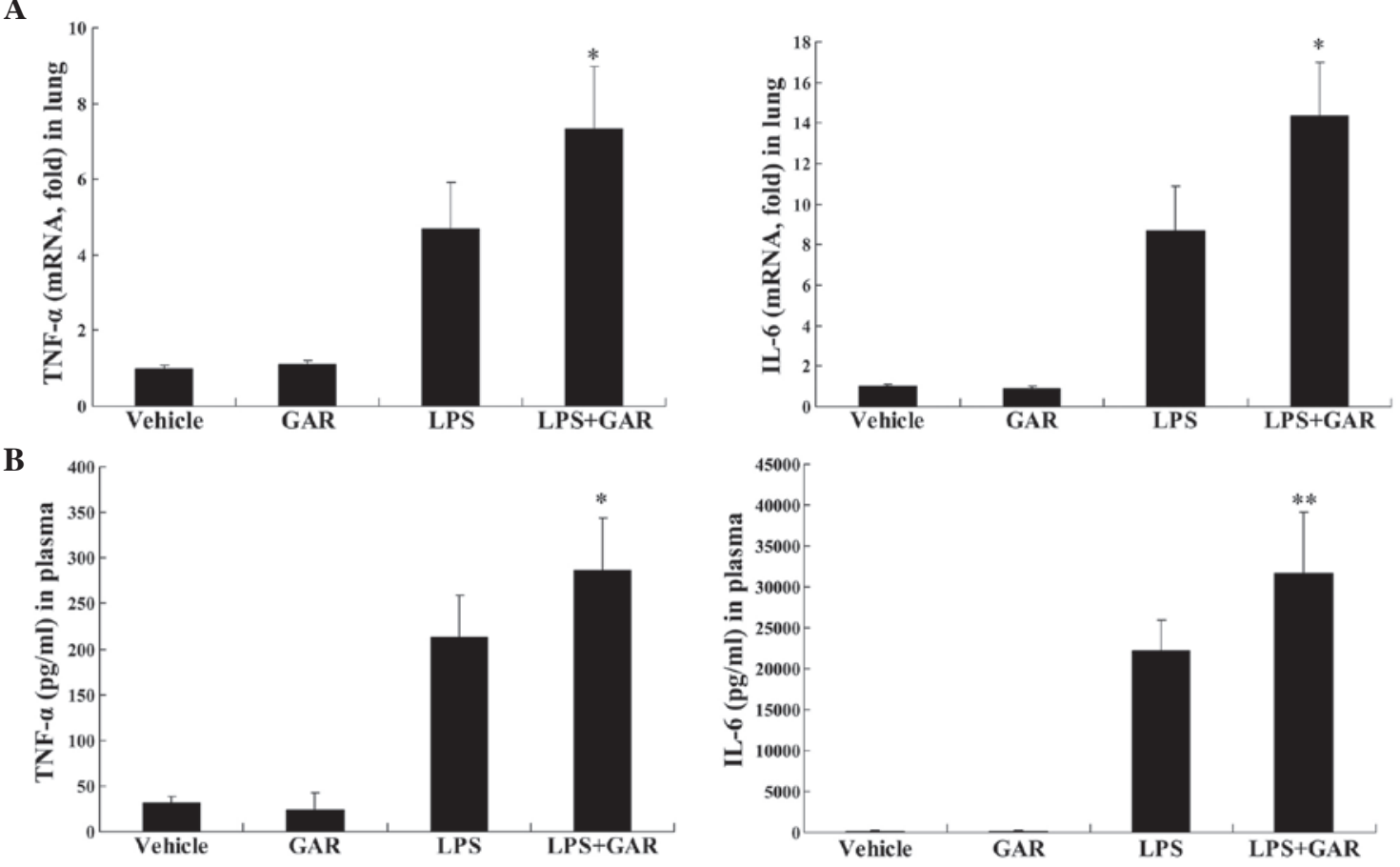

Figure 3. GAR promotes the expression of TNF- $\alpha$ and IL-6 in LPS-challenged mice. LPS-challenged mice were treated with vehicle or $10 \mathrm{mg} / \mathrm{kg}$ GAR The lung and plasma samples were harvested $18 \mathrm{~h}$ subsequent to LPS challenge. The (A) mRNA levels of TNF- $\alpha$ and IL-6 in lung tissue were determined by reverse transcription-quantitative polymerase chain reaction and the (B) protein levels of TNF- $\alpha$ and IL-6 in plasma were determined by enzyme-linked immunosorbent assay. Data are expressed as the mean \pm standard deviation, $n=8 .{ }^{*} \mathrm{P}<0.05,{ }^{* *} \mathrm{P}<0.01$ vs. LPS group. GAR, garcinol; TNF- $\alpha$, tumor necrosis factor- $\alpha$; IL-6, interleukin-6; LPS, lipopolysaccharide.

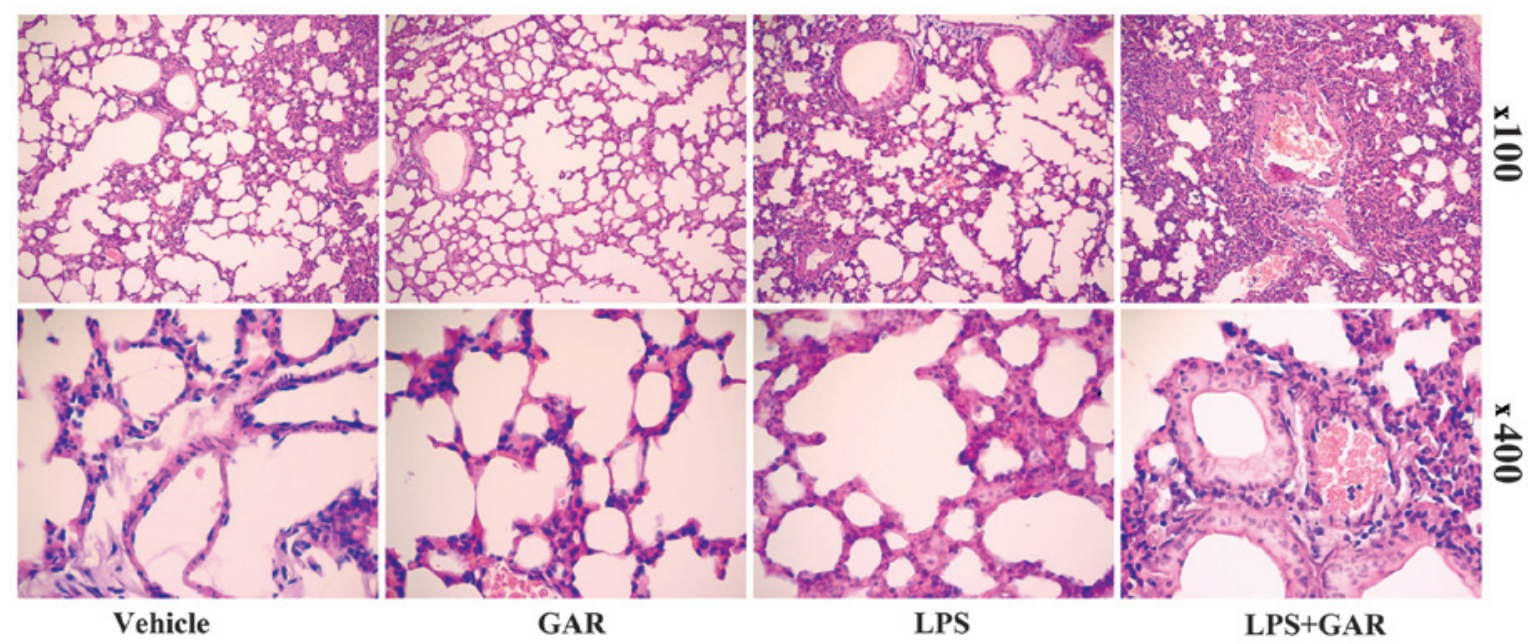

Figure 4. GAR exacerbates lung injury in LPS-challenged mice. LPS-challenged mice were treated with vehicle or $10 \mathrm{mg} / \mathrm{kg}$ GAR. The lung samples were harvested $18 \mathrm{~h}$ subsequent to LPS challenge and the lung sections were stained with hematoxylin-eosin for histopathological examination. Representative histological images of each group are presented (original magnification x100 and x400). GAR, garcinol; LPS, lipopolysaccharide.

$(100 \mu \mathrm{g} / \mathrm{l})$. The cells and the supernatants were harvested at $18 \mathrm{~h}$ subsequent to LPS challenge for further experiments.

Statistical analysis. All data from the experiments are expressed as the mean \pm standard deviation. Statistical significance was determined by Student's t-test for comparisons of two groups. Multi-group comparisons were performed using one-way analysis of variance for multiple comparisons among means, with Tukey's post-hoc test. Survival statistics were analyzed with a Kaplan-Meier curve and log-rank test.
Statistical analysis was conducted with SPSS 12.0 (SPSS, Inc., Chicago, IL, USA) and $\mathrm{P}<0.05$ was considered to indicate a statistically significant difference.

\section{Results}

Garcinol enhances LPS-induced inflammation in vitro. HATs catalyze the acetylation of histones and promote transcriptional activation (13), thus, inhibition of HATs by garcinol may result in the suppressed transcription of genes. However, 
unexpectedly, the present study demonstrated that garcinol (0.1-10 $\mu \mathrm{mol} / \mathrm{l})$ dose-dependently upregulated LPS-induced production of TNF- $\alpha$ and IL-6 proteins in RAW264.7 macrophages compared with LPS treatment alone (Fig. 1A and B). A similar effect was observed on the mRNA levels of TNF- $\alpha$ and IL- 6 following garcinol treatment, which were significantly increased compared with LPS treatment alone $(\mathrm{P}<0.05$; Fig. 1C and D).

Garcinol suppresses LPS-induced acetylation of NF- $\kappa$ B . $\mathrm{NF}-\mathrm{\kappa B}$ is a pivotal transcription factor in inflammation and its activity is enhanced by acetylation at Lys 310 in its p65 subunit (14). The present study demonstrated that LPS markedly stimulated the acetylation of p65 at Lys310, suggesting that acetylation of p65 may be involved in LPS-induced inflammation. By contrast, treatment with garcinol significantly attenuated LPS-induced p65 acetylation compared with LPS treatment only (Fig. 2), which may be caused by the inhibitory activity of garcinol on HATs.

Garcinol enhances LPS-induced production of pro-inflammatory cytokines in mice. In LPS-challenged mice, the present study demonstrated that treatment with garcinol significantly increased LPS-induced upregulation of TNF- $\alpha$ mRNA in lung tissue $(\mathrm{P}<0.05$; Fig. $3 \mathrm{~A})$ and TNF- $\alpha$ protein in plasma $(\mathrm{P}<0.05$; Fig. 3B) compared with LPS treatment only. Similarly, the mRNA $(\mathrm{P}<0.05$; Fig. $3 \mathrm{~A})$ and protein $(\mathrm{P}<0.01$; Fig. 3B) levels of IL-6 in LPS-insulted mice were significantly increased following garcinol treatment compared with LPS treatment alone. These data suggest that the HAT inhibitor, garcinol, may enhance LPS-induced inflammation in vivo.

Garcinol increased LPS-induced multi-organ injury and death in mice. Increased production of pro-inflammatory cytokines can result in more severe tissue damage. Consistent with the upregulated expression of TNF- $\alpha$ and IL-6 (Fig. 3), histopathological examination indicated that, compared with LPS treatment, the LPS-induced swelling of the alveolar walls and infiltration of leukocytes were further exacerbated by garcinol treatment (Fig. 4). Additionally, the plasma levels of ALT and BUN in LPS-exposed mice, used as markers for evaluating the lesions of the liver and kidneys, respectively, were significantly increased in the garcinol treated group compared with the LPS group $(\mathrm{P}<0.05$ and $\mathrm{P}<0.01$, respectively; Fig. $5 \mathrm{~A}$ and $\mathrm{B})$. To determine the final outcome of LPS-induced systemic inflammation, the difference in survival rate between LPS and LPS + garcinol-treated animals was compared. As demonstrated in Fig. 5C, compared with LPS treatment only, garcinol significantly shortened the survival time and increased the mortality of LPS-insulted mice $(\mathrm{P}<0.01)$.

\section{Discussion}

Garcinol, a polyisoprenylated benzophenone derivative of Garcinia indica fruit rind, was previously identified as a potent HAT inhibitor $(15,16)$. HATs catalyze the acetylation of histones, which is associated with opened chromatin structure and transcriptional activation $(17,18)$. Thus, inhibition of HATs by garcinol may result in suppressed transcription of genes. It was previously reported that garcinol
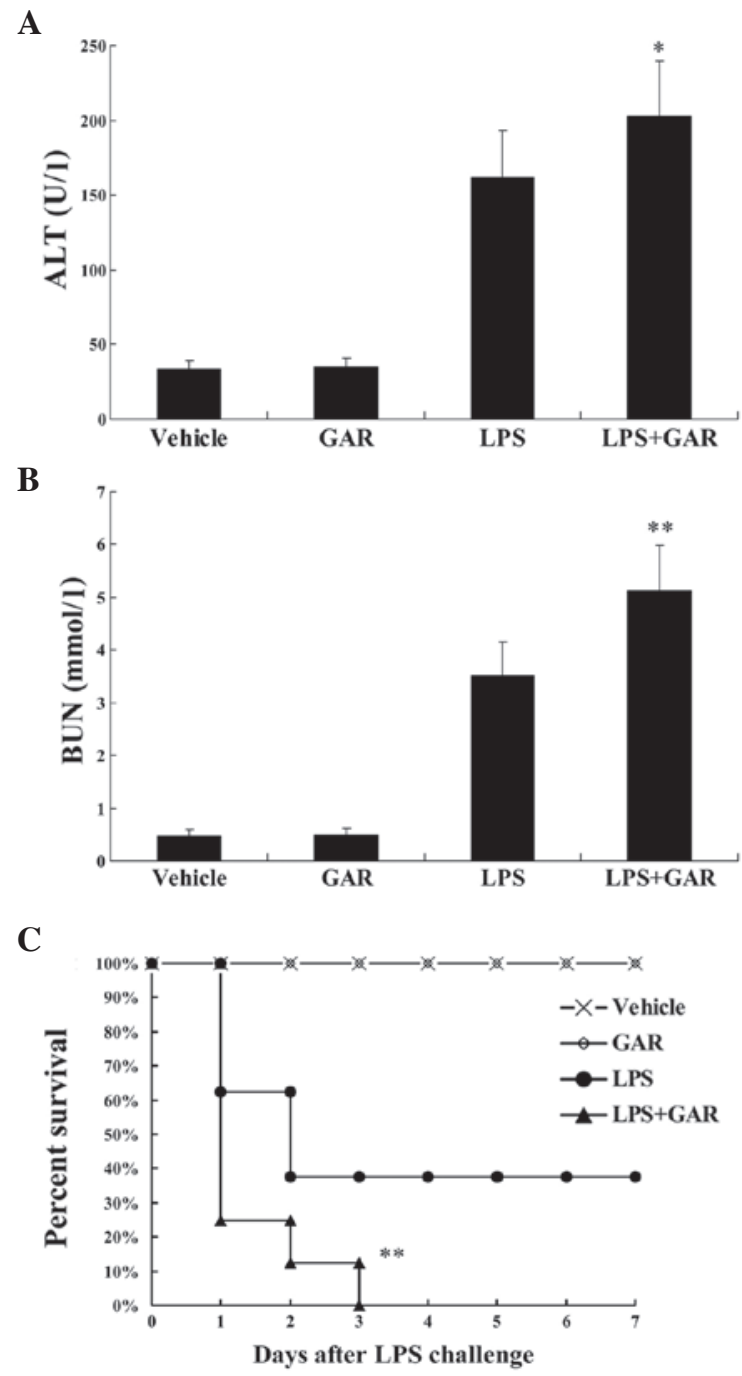

Figure 5. GAR increases the plasma levels of ALT and BUN, and reduces the survival rate of LPS-challenged mice. LPS-challenged mice were treated with vehicle or $10 \mathrm{mg} / \mathrm{kg}$ GAR. The lung and plasma samples were harvested $24 \mathrm{~h}$ subsequent to LPS challenge. The plasma levels of (A) ALT and (B) BUN were determined. Data are expressed as the mean \pm standard deviation, $n=8$. ${ }^{*} \mathrm{P}<0.05,{ }^{* *} \mathrm{P}<0.01$ vs. LPS group. (C) A different set of animals were allocated and the mortality of the mice was monitored every $6 \mathrm{~h}$. The percent survival rates are expressed as Kaplan-Meier curves $(n=20) .{ }^{* *} \mathrm{P}<0.01$ vs. LPS group. GAR, garcinol; ALT, alanine aminotransferase; BUN, blood urea nitrogen; LPS, lipopolysaccharide.

inhibits the expression of inducible nitric oxide synthase and cyclooxygenase-2 (COX-2) in LPS-stimulated macrophages, and TNF- $\alpha$-stimulated 3T3-L1 adipocytes $(17,19)$. Additionally, a previous study demonstrated that treatment with garcinol markedly inhibited cigarette smoke extract-stimulated COX-2 expression in human tracheal smooth muscle cells (19). Thus, garcinol appears to exhibit certain anti-inflammatory activities.

However, the present study unexpectedly demonstrated that garcinol promotes LPS-induced TNF- $\alpha$ and IL-6 production in vivo and in vitro. These effects were accompanied by enhanced organ damage and a reduced survival rate in LPS-challenged mice. Notably, it was previously reported that garcinol treatment did not alter the levels of TNF- $\alpha$ and IL-1 $\beta$ in LPS-stimulated leukocytes (20). Thus, it appears that garcinol may exhibit diverse effects on the expression of 
inflammatory genes and only suppresses inflammation under certain circumstances.

In contrast to HATs, HDACs catalyze the removal of the acetyl group from histones, which typically results in a condensed chromatin structure and transcriptional silencing (21). However, increasing evidence suggests that pharmacological inhibition of HDACs may suppress the expression of inflammatory genes and attenuate tissue injury in inflamed organs $(4,22,23)$. The observations of the present study were consistent with the previously reported anti-inflammatory actions of HDAC inhibitors, as garcinol HAT inhibitor exhibited the opposite effects on inflammation.

Although acetylation modification was originally observed in histones, and the enzymes controlling this modification were termed HATs and HDACs, it is now widely accepted that HATs/HDACs also catalyze the acetylation/deacetylation of certain non-histone proteins (6). In addition to histones, $\mathrm{NF}-\kappa \mathrm{B}$, a pivotal inflammation-associated transcription factor (24), can be acetylated at multiple lysine residues. It was previously reported that acetylation of the p $65 \mathrm{NF}-\kappa \mathrm{B}$ subunit at Lys310 or Lys221 increases the transcriptional activity or DNA binding affinity of NF- $\kappa \mathrm{B}(25,26)$, whereas, acetylation at Lys122 and Lys123 facilitated the relocation of NF- $\kappa$ B from the nucleus (27). Therefore, acetylation of p65 at discrete sites has distinct regulatory action on the functions of $\mathrm{NF}-\kappa \mathrm{B}$.

Other acetylation targets are also associated with the regulation of inflammation. High mobility group box 1 (HMGB1) is a chromatin protein that is secreted by activated macrophages as an inflammatory mediator $(28,29)$. Acetylation of HMGB1 promotes its translocation into the cytosol and facilitates its secretion (30,31). Additionally, acetylation of MKP-1, a negative regulator of the MAPK pathway, increases its phosphatase activity and interrupts the inflammatory signals transduced via the MAPK pathway $(4,32)$. Thus, acetylation modifications have positive and negative regulatory effects on inflammatory gene expression. The outcomes of HDAC or HAT inhibition in inflammation may be a result of the combined effects on these multiple targets.

In conclusion, the present study demonstrated that garcinol HAT inhibitor enhanced LPS-induced inflammation in vitro and in vivo. The observations of the current study, together with the anti-inflammatory properties of HDAC inhibitors, suggest that inhibition of deacetylation modulation may be correlated with suppressed inflammatory responses and alleviated tissue injury, however, the detailed molecular mechanisms underlying the pro-inflammatory effects of HAT inhibitors and the anti-inflammatory effects of HDAC inhibitors require further investigation. Thus, HDAC inhibitors, but not HAT inhibitors, may exert therapeutic effects in inflammatory disorders.

\section{Acknowledgements}

The current study was supported by grants from the Health and Family Planning Commission of Chongqing (grant no. 2012-2-035), the Natural Science Foundation of Chongqing (grant no. cstc2012jjA10041), the Hubei Provincial Department of education (grant no. D20144301) and the National Natural Science Foundation of China (grant no. 81370179).

\section{References}

1. Kotas ME and Medzhitov R: Homeostasis, inflammation, and disease susceptibility. Cell 160: 816-827, 2015.

2. Ehrentraut SF and Colgan SP: Implications of protein post-translational modifications in IBD. Inflamm Bowel Dis 18: 1378-1388, 2012.

3. Choudhary C, Weinert BT, Nishida Y, Verdin E and Mann M: The growing landscape of lysine acetylation links metabolism and cell signalling. Nat Rev Mol Cell Biol 15: 536-50, 2014

4. Jeong Y, Du R, Zhu X, Yin S, Wang J, Cui H, Cao W and Lowenstein CJ: Histone deacetylase isoforms regulate innate immune responses by deacetylating mitogen-activated protein kinase phosphatase-1. J Leukoc Biol 95: 651-659, 2014.

5. Chi H and Flavell RA: Acetylation of MKP-1 and the control of inflammation. Sci Signal 1: pe 44, 2008.

6. Spange S, Wagner T, Heinzel T and Krämer $\mathrm{OH}$ : Acetylation of non-histone proteins modulates cellular signalling at multiple levels. Int J Biochem Cell Biol 41: 185-198, 2009.

7. Hu X, Yu Y, Eugene Chin Y and Xia Q: The role of acetylation in TLR4-mediated innate immune responses. Immunol Cell Biol 91: 611-614, 2013

8. Gräff J and Tsai LH: Histone acetylation: Molecular mnemonics on the chromatin. Nat Rev Neurosci 14: 97-111, 2013.

9. Royce SG and Karagiannis TC: Histone deacetylases and their inhibitors: New implications for asthma and chronic respiratory conditions. Curr Opin Allergy Clin Immunol 14: 44-48, 2014.

10. Maddox SA, Watts CS, Doyère V and Schafe GE: A naturally-occurring histone acetyltransferase inhibitor derived from Garcinia indica impairs newly acquired and reactivated fear memories. PLoS One 8: e54463, 2013.

11. Ye X, Yuan L, Zhang L, Zhao J, Zhang CM and Deng HY: Garcinol, an acetyltransferase inhibitor, suppresses proliferation of breast cancer cell line MCF-7 promoted by 17 $\beta$-estradiol. Asian Pac J Cancer Prev 15: 5001-5007, 2014.

12. Schefe JH, Lehmann KE, Buschmann IR, Unger T and Funke-Kaiser H: Quantitative real-time RT-PCR data analysis: Current concepts and the novel 'gene expression's CT difference' formula. J Mol Med (Berl) 84: 901-910, 2006.

13. Gong F and Miller KM: Mammalian DNA repair: HATs and HDACs make their mark through histone acetylation. Mutat Res 750: 23-30 2013.

14. Ghizzoni M, Haisma HJ, Maarsingh H and Dekker FJ: Histone acetyltransferases are crucial regulators in NF- $\mathrm{BB}$ mediated inflammation. Drug Discov Today 16: 504-511, 2011.

15. Semwal RB, Semwal DK, Vermaak I and Viljoen A: A comprehensive scientific overview of Garcinia cambogia. Fitoterapia 102: 134-148, 2015

16. Padhye S, Ahmad A, Oswal N and Sarkar FH: Emerging role of Garcinol, the antioxidant chalcone from Garcinia indica Choisy and its synthetic analogs. J Hematol Oncol 2: 38, 2009.

17. Liao CH, Sang S, Liang YC, Ho CT and Lin JK: Suppression of inducible nitric oxide synthase and cyclooxygenase-2 in downregulating nuclear factor-kappa B pathway by Garcinol. Mol Carcinog 41: 140-149, 2004.

18. Yang CM, Lee IT, Lin CC, Yang YL, Luo SF, Kou YR and Hsiao LD: Cigarette smoke extract induces COX-2 expression via a PKCalpha/c-Src/EGFR, PDGFR/PI3K/Akt/NF-kappaB pathway and p300 in tracheal smooth muscle cells. Am J Physiol Lung Cell Mol Physiol 297: L892-L902, 2009.

19. Hsu CL, Lin YJ, Ho CT and Yen GC: The inhibitory effect of pterostilbene on inflammatory responses during the interaction of 3T3-L1 adipocytes and RAW 264.7 macrophages. J Agric Food Chem 61: 602-610, 2013.

20. Sailhamer EA, Li Y, Smith EJ, Shuja F, Shults C, Liu B, Soupir C, deMoya M, Velmahos G and Alam HB: Acetylation: A novel method for modulation of the immune response following trauma/hemorrhage and inflammatory second hit in animals and humans. Surgery 144: 204-216, 2008.

21. Seto E and Yoshida M: Erasers of histone acetylation: The histone deacetylase enzymes. Cold Spring Harb Perspect Biol 6: a018713, 2014.

22. Cantley MD and Haynes DR: Epigenetic regulation of inflammation: progressing from broad acting histone deacetylase (HDAC) inhibitors to targeting specific HDACs. Inflammopharmacology 21: 301-307, 2013.

23. Chen HY, Li L and Fu ZJ: Histone deacetylase inhibitors trichostatin A and suberoylanilide hydroxamic acid attenuate ventilator-induced lung injury. Pharmazie 69: 55-59, 2014. 
24. Hoesel B and Schmid JA: The complexity of NF- $\mathrm{BB}$ signaling in inflammation and cancer. Mol Cancer 12: 86, 2013.

25. Rothgiesser KM, Erener S, Waibel S, Lüscher B and Hottiger MO: SIRT2 regulates $N F-\kappa B$ dependent gene expression through deacetylation of p65 Lys310. J Cell Sci 123 4251-4258, 2010.

26. Zhang Y, Qiu J, Wang X Zhang Y and Xia M: AMP-activated protein kinase suppresses endothelial cell inflammation through phosphorylation of transcriptional coactivator p300. Arterioscler Thromb Vasc Biol 31: 2897-2908, 2011.

27. Chen LF and Greene WC: Shaping the nuclear action of NF-kappaB. Nat Rev Mol Cell Biol 5: 392-401, 2004.

28. Lee SA, Kwak MS, Kim S and Shin JS: The role of high mobility group box 1 in innate immunity. Yonsei Med J 55: 1165-1176, 2014.
29. Erlandsson Harris $\mathrm{H}$ and Andersson U: Mini-review: The nuclear protein HMGB1 as a proinflammatory mediator. Eur J Immunol 34: 1503-1512, 2004.

30. Andersson U, Antoine DJ and Tracey KJ: The functions of HMGB1 depend on molecular localization and post-translational modifications. J Intern Med 276: 420-424, 2014.

31. Dhupar R, Klune JR, Evankovich J, Cardinal J, Zhang M, Ross M, Murase N, Geller DA, Billiar TR and Tsung A: Interferon regulatory factor 1 mediates acetylation and release of high mobility group box 1 from hepatocytes during murine liver ischemia-reperfusion injury. Shock 35: 293-301, 2011.

32. Chuang YF, Yang HY, Ko TL, Hsu YF, Sheu JR, Ou G and Hsu MJ: Valproic acid suppresses lipopolysaccharide-induced cyclooxygenase-2 expression via MKP-1 in murine brain microvascular endothelial cells. Biochem Pharmacol 88: 372-383, 2014. 